\title{
Research on the Adaptive Design of Song Typeface Under the Electronic Screen
}

\author{
Zhixiong Huang ${ }^{1, *}$ Xiaozhen Huang ${ }^{1}$
}

${ }^{1}$ Fuzhou University, Fuzhou, Fujian 350000, China

*Corresponding author.Email: 38501093@qq.com

\begin{abstract}
Objective: The paper explores the use of adaptability theory to optimize the feasibility of Song typeface under the electronic screen. Method: It starts from the adaptability theory and maps to the adaptive design of fonts, analyzes the law of the evolution of fonts and the characteristics of electronic screens, and draws lessons from the design experience of boldface under the electronic screen. Guided by the adaptive design theory, and under the premise of not destroying the essential characteristics of Song typeface, the adaptive design strategy of Song typeface under the electronic screen is proposed. Finally, it takes the font interface of "Xue Xi Qiang Guo" APP as an example to start the design and verify the feasibility of practice. Conclusion: In the optimization design of Song typeface under the electronic screen, it is necessary to emphasize the human experience as the starting point, and use the theory of applicability to guide the redesign and readjustment of Song typeface's strokes to enhance the user's comfort level of reading experience.
\end{abstract}

Keywords: electronic screen, adaptive design, Song typeface, reading experience

\section{INTRODUCTION}

With the popularization of smart phones, screen display watches, and tablet PC (tablet personal computer), electronic screens have gradually surpassed traditional paper media as the mainstream carrier of information transmission. The emergence of new media carriers always points new directions and new requirements for font design. Song typeface is a classic that can afford Chinese fonts. However, due to the coexistence of Song typeface with typography, the charm of fonts since its birth makes it difficult for Song typeface to adapt to electronic screens. The adaptation of Song typeface to electronic technology has not found a way of innovation. Compared with boldface, the derived font library of Song typeface is still quite lacking. Therefore, this paper will introduce the concept of adaptive design, analyze the adaptive evolution of fonts in each era, pay attention to readers' reading experience, and give some inspiration to the design of Song typeface under the electronic screen, so as to make up for the lack of font derivation of Song typeface on the screen display.

\section{THE ADAPTABILITY THEORY OF FONTS}

Adaptability theory originated from biology. It first appeared in Darwin's book of "Origin of Species", and because of its extensive characteristics, it has expanded to various academic fields such as ethnology, geography, and philosophy. Adaptability refers to the ability of an individual to respond appropriately to the constraints or changes of the environment in the living environment, so that a man and the environment can still maintain a harmonious state. [1] Therefore, before extending the adaptability theory to font design, the font design process is divided into four components: (1) people; (2) fonts; (3) media; (4) design. While driven by the four elements, adaptability exists between the design process, where people are the foothold, and design is the central link in the adaptability process. In order to meet their own reading needs, people can adapt fonts and media through design. The adaptability process of fonts is diverse, facing a dynamically changing medium, and people's reading needs change with the change of the medium. When fonts appear unsuitable, they are constantly adjusted through design. This is also the motivation for fonts to remain vigorous. Therefore, taking into account the adaptation characteristics between the font and the medium, the human reading experience, and the adaptability of the design play a very important role in the adaptive design of the font.

\section{THE ADAPTIVE EVOLUTION OF FONTS}

From the archeological discovery of Jiahu's inscribed symbols, Chinese characters have gone through a history of more than 8,000 years. From the recorded legend of Cangjie's creating characters to the oracle bones found in archaeological discovery with characters evidence, the font of Chinese characters is 
closely related to its carrier. The Oracle Bones from the Yin Ruins are the earliest and relatively complete recorded characters. On the tortoise shells and animal bones as its carrier, the strokes are mostly straight lines without change, and even the curved lines are carved by short straight lines. During the Shang and Zhou dynasties, with the popularity of bronze vessels, the inscriptions on ancient bronze objects (inscriptions) attached to the rounded bronze ware raised the curtain. In the late Western Zhou Dynasty, bamboo slips evolved into the main character carrier. The large seal script that was easy to portray was conceived and born, and its uniform and distinct shapes laid the foundation for the subsequent Chinese characters. After the Qin Dynasty unified the Six Kingdoms, the small seal script became the only universal font. Compared with the large seal script, it made a qualitative leap in shape and quantity. With the invention of papermaking, the character carrier gradually changed from bamboo slips to paper. The clerical script is the creation of characters by Cheng Miao based on the functionality of the characters. It is the watershed between ancient and modern characters. The clerical script is based on the evenly rounded lines of the small seal script strokes, and the pictograph is changed to straight and square strokes. It is a typeface for the convenience of writing and the large demand for text. After Cai Lun improved papermaking in the Eastern Han Dynasty, writing on paper became popular. During this period, due to the characteristics of paper and the randomness of the smart changes of the brush, the calligraphy culture was on the stage of history. Based on the clerical script, cursive script, running script and regular script were derived. In the heyday of the Tang Dynasty, the demand for books increased sharply, and block printing became an inevitable invention under the trend of the times. Because of its sparse strokes, regular script basically satisfies the comfort of paper reading, it has been used from written text to printed text, and it is also a common font for early block printing. And Song typeface is a font created by the continuous improvement of printing technology and the structure of characters strokes. Since then, the design of printed fonts has been continuously exploring the creation of characters in accordance with the comfort of reading and the convenience of printing. After entering the great digital age, electronic screens have entered people's lives as a brand-new character carrier. Today, people have all become "screen citizens", so the experience of reading text on electronic screens is particularly important. The characteristics of the boldface itself have natural advantages on the electronic screen, and on the basis of the structural characteristics of the boldface, many boldface fonts have been created, such as the typical Fang Zheng You Hei Ti, Microsoft Ya Hei and so on.
From the above point of view, when a new medium appears, the adaptation of fonts becomes inadaptation. The evolution of fonts is a dynamic cycle from inadaptation to adaptation. Adaptability can also be regarded as one of the essential characteristics of fonts. Fonts that can adapt to the current medium become the mainstream fonts of this period, and those that can't adapt will inevitably be ignored. This is also consistent with Darwin's evolutionary theory of "survival of the fittest". For fonts, how to maintain vitality is particularly important to the interpretation of current popular media.

\section{THE CHARACTERISTICS OF ELECTRONIC SCREENS AS CHARACTER CARRIER}

In recent years, from LED screens to watches, electronic screens have penetrated into all areas of life in various forms. The more and more intelligent life, basic necessities, food, housing and transportation can be basically satisfied on the screen of the mobile phone. Traditional paper is no longer the only carrier of characters, and electronic screens have long become an important medium. This brand new character carrier is different from any carrier in the past. Its characteristics are:

\section{A. Pixelate}

Grid-shaped pixels are the smallest unit of electronic display screens. The character and even images presented on the screen are composed of countless grid-shaped pixels. This feature determines how to improve the recognizability and accuracy is an important design experiment direction for screen display fonts.

\section{B. Self-illumination}

The current-mode self-luminous screen is one of the main features that differentiates itself from paper media. As a self-luminous character carrier, the brightness of the screen causes the thinner strokes in the font to be eroded to varying degrees, directly affecting the visual senses and forming a different experience feeling of reading.

\section{Small font size}

Mobile phones are the most commonly used electronic screen carriers in today's society. The primary external function of mobile phones is portability, therefore, electronic screens are different from traditional paper media. The display size is limited by the size of mobile phones, which directly affects the font and font size on the electronic screen. It is difficult to show the details of the font stroke structure on a small screen. Therefore, the use of electronic screens as the carrier to spread fonts requires more attention to the screen resolution, viewing distance, display color, 
brightness, contrast and other elements. For Song typeface, regular script, handwritten fonts, etc., which have good visual effects on traditional paper media, they may not be suitable for presentation on electronic screens.

\section{ADAPTIVE DESIGN OF BOLDFACE BASED ON READING EXPERIENCE}

The concept of experience comes from psychology and refers to the internal reaction of the subject to the stimulation of the object. [2] The so-called reading experience is actually the process by which readers read the text presented through the media and gain experience and inspiration. Characters are gradually transferred from paper copying to electronic screens, and readers' reading of long texts has evolved to obtain them from electronic screens. Boldface's various design attempts under the electronic screen are centered on the reader, and how to display the best effect on the electronic screen and obtain a comfortable experience as the problem-oriented. You Hei Ti puts forward the general design principle of "making text more kind and making reading more comfortable" for screen reading. Since the screen is composed of countless small squareshaped pixels, it is more advantageous for straight line display. Therefore, western fonts with boldface and sans serifs are widely used on electronic screens. Boldface itself has the characteristics of equal-width straight line and sans serif decoration. Compared with Song typeface, the font designer has designed the matching boldface font according to the characteristics of electronic screens, and has achieved good response. For example, the most widely used Windows and Mac systems, popular APPs such as WeChat, Netease Cloud and MicroBlog are all based on the boldface's stroke structure to design and create characters.

Regarding how to satisfy readers' reading experience, boldface makes the following attempts: first, in "Fig. 1", it splits the stroke structure of boldface, and the thickness of the strokes is almost the same. Compared with Song typeface, although it lacks rhythm, it is more fluent in reading. Second, "Fig. 2" selects several typical screen boldface fonts. It can be found that in the processing of stroke structure, it has adopted a compromise route, and reasonably set the density and literal size of the Zhonggong ("中宫"). It not only guarantees the better resolution of the characters in small fonts, but also avoids the looseness of the Zhonggong caused by the too large font size, the evacuation of the frame structure, and the less detailed processing. It retains the stroke contour features of the character itself as much as possible, thereby improving the reading comfort of readers. Third, in the processing of horizontal and vertical strokes, it removes visual impurities and ghosting in the text display on the electronic screen. The position processing of the entire text distribution prevents uneven distribution of blank size and overlap and conglutination between strokes, preventing the literal effect of visually uneven thickness. It makes the black, white and grey formed after the overall layout of the text more pure, arousing the interest of reading.

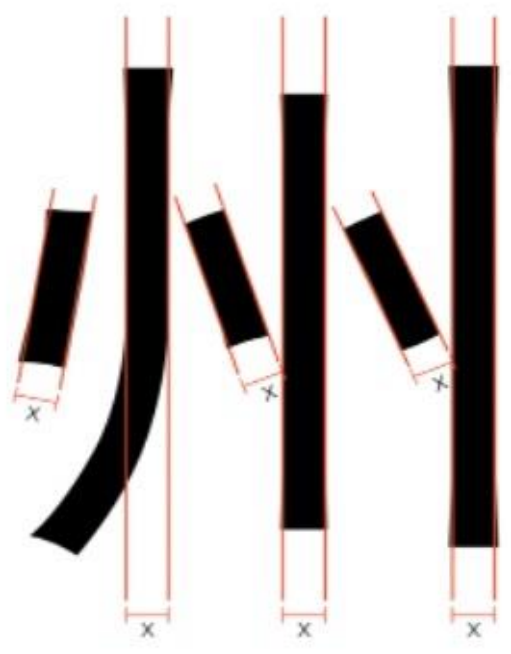

Fig. 1. Strokes in boldface.
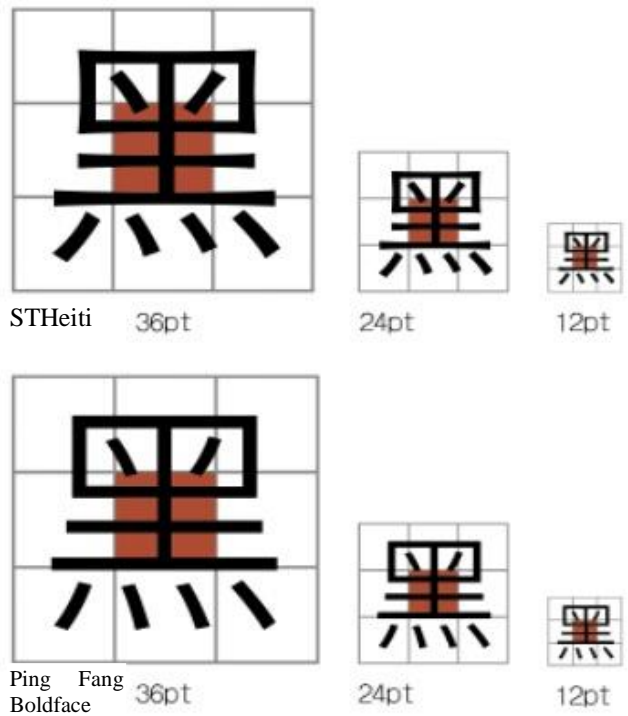

Fig. 2. Different font weights in the boldface's Zhonggong.

\section{THE ADAPTIVE DESIGN STRATEGY OF SONG TYPEFACE UNDER THE ELECTRONIC SCREEN}

\section{A. The mutual adaptability of Song typeface and the media}

From the Song Dynasty, Song typeface had an indissoluble bond with printing. With the prevalence of printing and publishing, Song typeface also began to 
derive, and straight strokes were extracted from regular scripts to standardize fonts. In the Ming Dynasty, characters of Song typeface increased the horizontal and vertical ratio in the structure of the strokes, and the turning points were mostly decorated with triangles to fit the current printing effect and facilitate carving and recognition. Developed to modern times, more convenient lead type typesetting was conceived, and Song typeface made corresponding adjustments to the strokes. The final Song typeface character is the sum of the evolution of Song typeface in various periods. It has to say that the perfect presentation of Song typeface is now a result of millennial exploration and integration with printing technology. Song typeface extracts strokes from calligraphy regular script, condensing Chinese calligraphy culture. The author believes that Song typeface is one of the shining pearls of Chinese national culture. Since its birth, Song typeface has occupied a mainstream position and has influenced generations of Chinese people.

The adaptability between Song typeface and the media is a long-term process. Although Song typeface has a strong adaptability in the printing media, it also appears inadaptability in the face of emerging media. The frame structure of Song typeface's strokes evolved from regular script. Song typeface's horizontal and vertical strokes have a strict fixed ratio, and there are triangle serifs in the first and last strokes of a character. The small font size of Song typeface is easy to overlook this detail, and the strokes are in a straight line visually. The characteristics of the horizontally thin and vertically thick of Song typeface are clearly displayed on the paper medium and are readable. However, on the electronic screen, between the direct interlock of strokes, blurs and ghosting appear with the reflection of light and shadow, and at the same time, the thinner strokes appear erosion to different degrees. The readability is poor, and the reading of long texts is also easy to cause fatigue.

\section{B. The adaptive design of Song typeface under the electronic screen}

The adaptability of Song typeface under the electronic screen is essentially the design of Song typeface to adapt to the reading needs of people on the electronic screen, which is an active redesign. The version scholars define Song typeface characters as "horizontally flat, vertically straight, horizontally thin, vertically thick, with edges and corners in the first and last strokes, square fonts, and starched strokes."[3] On the premise of not changing the rhyme characteristics of Song typeface, and grasping the scope of adaptation of Song typeface design, by absorbing boldface's improved design experience for the screen, a preliminary plan for the redesign of Song typeface is proposed:
1) Redesign of font strokes: All Chinese characters are made up of strokes. In "Introduction to Science of Chinese Characters Formation", Wang Ning mentioned that the strokes of Chinese characters can be divided into eight types: horizontal stroke, vertical stroke, leftfalling stroke, right-falling stroke, turning stroke, dot stroke, raising stroke, and vertical hook stroke. Therefore, the various strokes of Song typeface are disassembled, reorganized, and integrated ("Table I"). The horizontally thin and vertically thick stroke is one of the characteristics that distinguish Song typeface from other fonts. On the premise of retaining this feature, it appropriately adjusted the "vertical" strokes ("Fig. 3"). After repeated comparisons, the original horizontal and vertical thickness is adjusted from 1:3 to 1.6:2, which is the relatively comfortable proportional relation on the electronic screen. Serif is another feature that distinguishes Song typeface from most fonts. The strokes of Song typeface with edges and corners are difficult to adapt to electronic screens. On the basis of drawing on the end of the boldface's "trumpet mouth" strokes, it smooths the serif of Song typeface, and is no longer eye-catching at the turning point, weakening the rhythm of the font, and making it more standardized and stylized. With this change, the Song typeface has a clear thickness difference between horizontal and vertical strokes on the large font size display, which retains the characteristics of Song typeface; in the display of small font sizes, the gap becomes smaller and the details are weakened, and the recognizability of the character has been significantly improved, ensuring the recognizability of fonts under various font sizes, and increasing the scope of application of Song typeface. 
TABLE I. STROKE OPTIMIZATION OF THE SONG TYPEFACE'S SCREEN DISPLAY

\begin{tabular}{|c|c|c|c|}
\hline Strokes & Song typeface & Optimize & $\begin{array}{l}\text { Optimize } \\
\text { screen display } \\
\text { of Song } \\
\text { typeface }\end{array}$ \\
\hline Horizontal stroke & & $\begin{array}{l}\text { The serif decoration in the first and last strokes } \\
\text { is weakened, and the strokes are appropriately } \\
\text { thickened }\end{array}$ & \\
\hline Vertical stroke & & $\begin{array}{c}\text { The serif decoration in the first and last strokes } \\
\text { is weakened, and the strokes are appropriately } \\
\text { thickened }\end{array}$ & \\
\hline Left-falling stroke & & $\begin{array}{l}\text { The serif in the first stroke is weakened, and } \\
\text { the last stroke is thickened }\end{array}$ & \\
\hline Right-falling stroke & & $\begin{array}{l}\text { The first stroke is thickened appropriately, and } \\
\text { the last stroke is transited smoothly }\end{array}$ & \\
\hline Turning stroke & & $\begin{array}{l}\text { The overlapping part of the intersection is } \\
\text { appropriately weakened, and the ratio of } \\
\text { horizontal and vertical is } 1.6: 2\end{array}$ & \\
\hline Dot stroke & & $\begin{array}{c}\text { The first stroke is thickened appropriately, and } \\
\text { the last stroke is properly uprightly and } \\
\text { foursquare handled }\end{array}$ & \\
\hline Raising stroke & & $\begin{array}{l}\text { The serif in the first stroke is weakened, and } \\
\text { the last stroke is thickened }\end{array}$ & \\
\hline Vertical hook stroke & & $\begin{array}{c}\text { The last stroke is appropriately smoothly } \\
\text { transited }\end{array}$ & \\
\hline
\end{tabular}

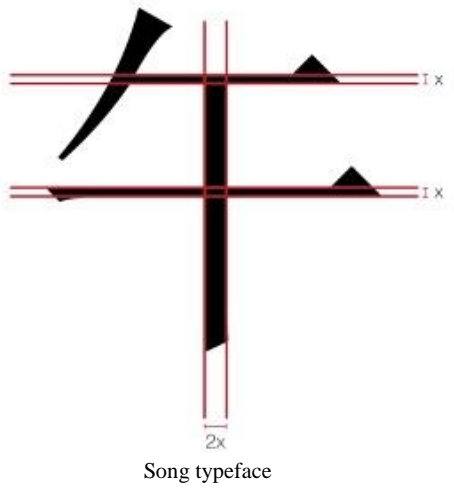

Fig. 3. Adjusting the stroke thickness ratio of Song typeface's screen display.

2) Re-adjustment of font structure: The term Zhonggong comes from calligraphy. The fifth grid from the left cis-position is the Zhonggong, which is also the most important grid for the structure of Chinese characters. Therefore, the effects of different sizes of the Zhonggong are also different. It takes the Chinese character Sudoku as an example, in "Fig. 4", the Sudoku is a regular and evenly divided Sudoku. The Sudoku in "Fig. 5" of the same size enlarges the middle grid. Through the visual changes of Sudoku from large to small, it can be seen that the conventional Sudoku

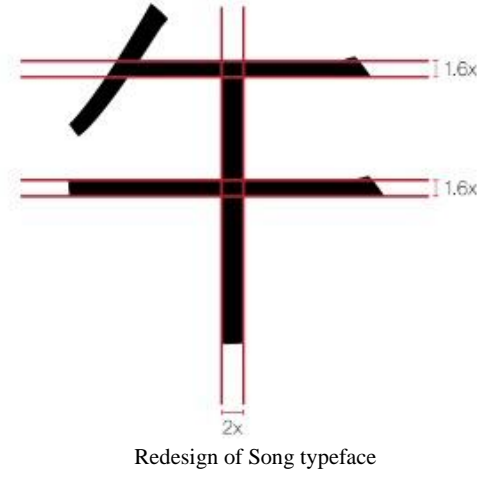

gives people a feeling of restraint and crowdedness, while the expansion of the middle space of the Sudoku is more balanced. Increasing the Zhonggong of font size helps to improve the recognition of the text on the electronic display screen, so that the font is still legible under different font sizes, and the comfortable reading of the text in terms of overall layout can be improved. But it still needs to consider what proportion of Sudoku is more suitable. Therefore, it places the Song typeface character "中" in the Sudoku of 1:1 and 1:1.5 in turn ("Fig. 6"). Repeated experiments and comparisons 
show that Sudoku of $1: 1$ and $1: 1.2$ are visually too compact and slender, while the Zhonggong of Sudoku of $1: 1.4$ and $1: 1.5$ are too loose, giveing people a cumbersome visual fatigue. And the Sudoku of $1: 1.3$ is relatively moderate in both large and small font sizes. This method is also applied to the screen display Song typeface ("Fig. 7"), the font on the left is traditional printed Song typeface, and the font on the right deliberately enlarges the Zhonggong according to the ratio of 1:1.3. This design makes the $B$ font look more breathable when displayed on the screen. Compared with the traditional Song typeface with the same font size, it is visually more comfortable and will not be visually tired due to long text reading.
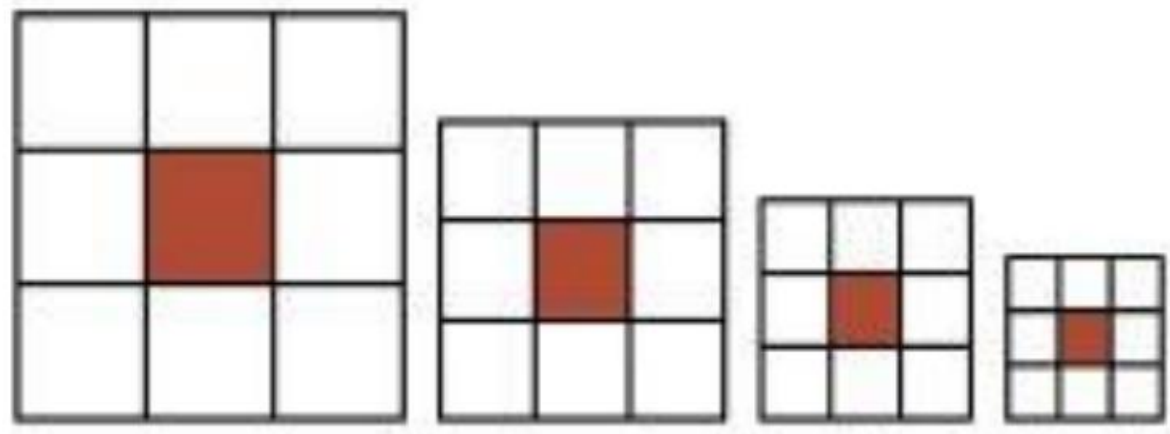

Fig. 4. Visual effects of different sizes of Zhonggong.
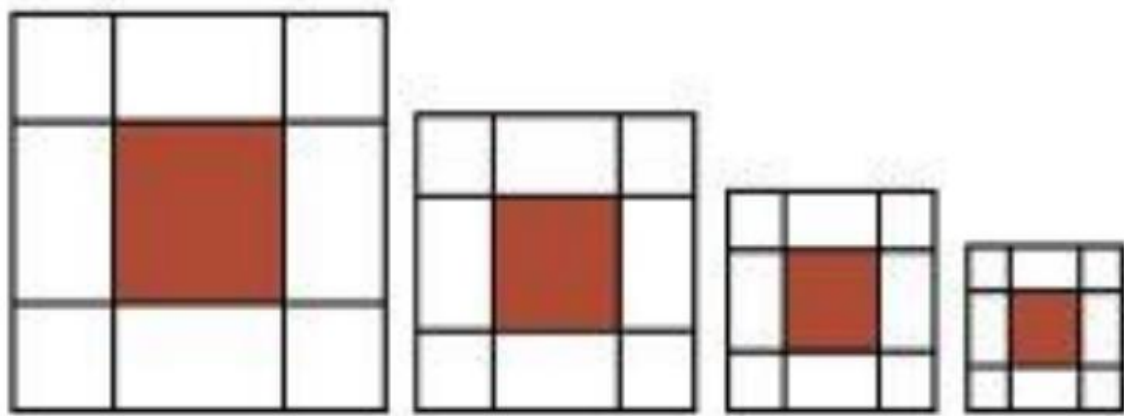

Fig. 5. Visual effects of different sizes of Zhonggong.
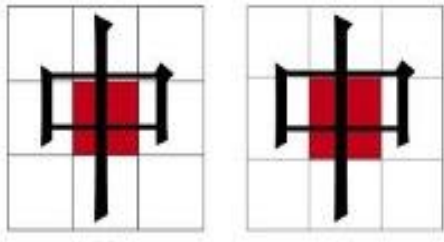

$1: 1.1$

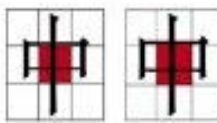

$1: 1$

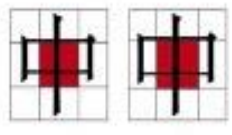

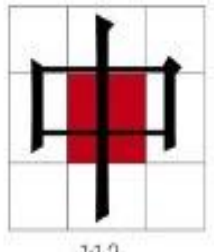

17.2

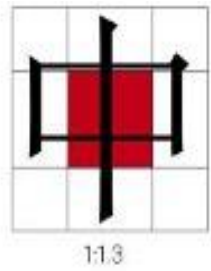

$1: 1.5$

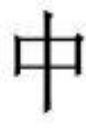

$1: 1,1$
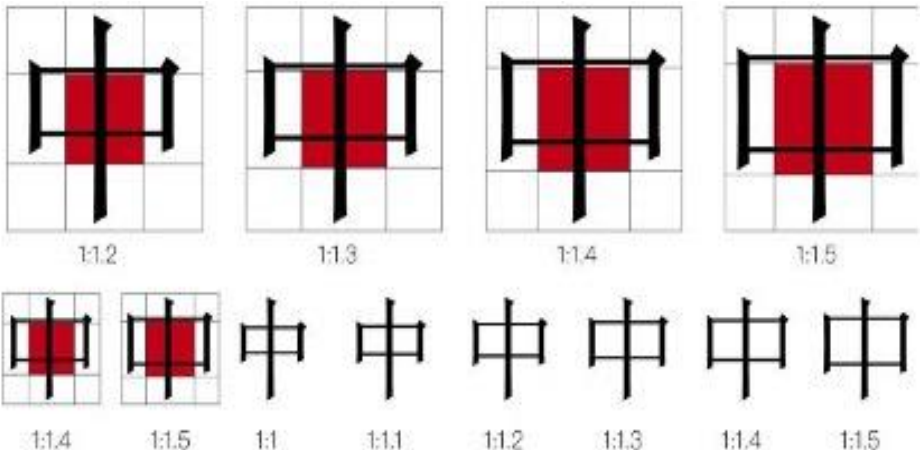

$1: 12 \quad 1: 1.3 \quad 1: 1.4$

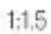

Fig. 6. Visual effects of the "中" character in different proportions of Zhonggong. 


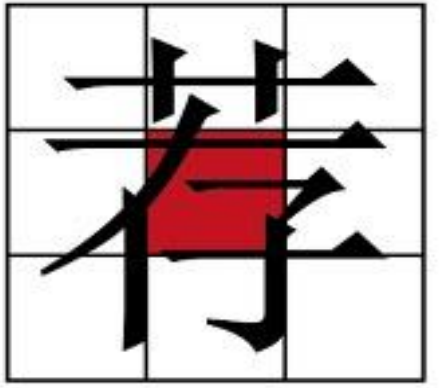

Song typeface

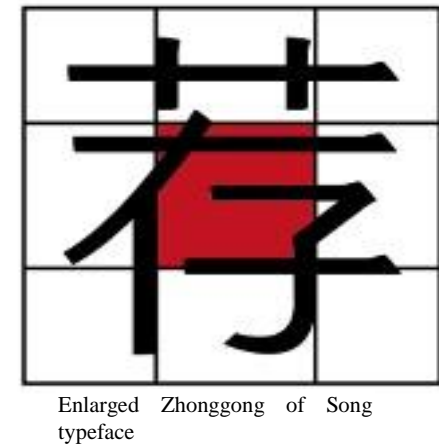

typeface

Fig. 7. The visual effect of optimizing screen display Song typeface

Mobile phones, tablet PC, and computers are electronic media that readers often read, but due to the small screens, the text displayed is usually small with mostly long text. In order to better improve the reading experience of Song typeface on the electronic screen, it is necessary to explore the layout between strokes and characters. For a single character, the parallel and intersection between the strokes need to be meticulously considered. On the basis of not changing the font size, the distance between the strokes should be properly extended. The intersections of strokes are often darker, so a soft transition is needed, and the intersections should be appropriately thinned. The screen is composed of pixels, which is different from the spontaneous light of other carriers. As a result, small font characters, especially those below $12 \mathrm{pt}$, are easy to generate ghosting and mottling of multi-stroke characters in the arrangement of long text, as well as blurring and erosion of characters with fewer strokes. Therefore, the font can't be adjusted in isolation, and it should also be placed in a long text to control the relationship between font strokes macroscopically. From this, it can help readers have a more comfortable reading experience for long texts. In addition, when designing the layout of Chinese characters, compared with the design of English fonts, proper handling of the relationship between the components of each Chinese character should also be considered. More than 50,000 Chinese characters embody the wisdom of the Chinese nation, which can be divided into two categories: monomeric characters and compound characters. Among them, the compound characters also include left-right structure, left-middle-right structure, upperlower structure, upper-middle-lower structure, halfenclosed structure, and full-enclosed structure ("Table II"). It summarizes the optimization of these seven types of glyph structures. However, the specific problems of specific issues should be analyzed differently. The structure of Chinese characters is not a mechanical copy. When a specific Chinese character is encountered, it should be adjusted in detail under the optimization of the large framework. 
TABLE II. THE FONT STRUCTURE OPTIMIZATION OF THE SCREEN DISPLAY SONG TYPEFACE

\begin{tabular}{|c|l|l|c|}
\hline Font structure & Song typeface & \multicolumn{1}{c|}{$\begin{array}{c}\text { Optimize } \\
\text { display of Song } \\
\text { typeface }\end{array}$} \\
\hline $\begin{array}{c}\text { Monomeric characters } \\
\text { Left-right structure }\end{array}$ & It has appropriate typeface with full font \\
\hline structure & $\begin{array}{l}\text { On the basis of full font, the area is allocated according } \\
\text { to the strokes of the two parts }\end{array}$ & $\begin{array}{l}\text { It allocates the area according to the strokes of the three } \\
\text { parts, pays attention to the stability of the middle part, } \\
\text { and balances the left and right parts }\end{array}$ \\
\hline $\begin{array}{c}\text { Upper-lower structure } \\
\text { structure }\end{array}$ & $\begin{array}{l}\text { It allocates the area according to the strokes of the } \\
\text { upper and lower parts, appropriately compacts the upper } \\
\text { part, relaxes the lower part, and avoids white space and } \\
\text { being too even }\end{array}$ & $\begin{array}{l}\text { It allocates the area according to the strokes of the three } \\
\text { parts, pays attention to the align center of the three } \\
\text { parts, and properly distinguishes the width }\end{array}$ & $\begin{array}{l}\text { The feature of incompletely closed glyph structure } \\
\text { requires two parts to be appropriately close, tight inside } \\
\text { and loose outside }\end{array}$ \\
\hline $\begin{array}{c}\text { Full-enclosed structure } \\
\text { The feature of fully-enclosed glyph structure requires } \\
\text { the peripheral components to be generous, leaving } \\
\text { space for internal components }\end{array}$
\end{tabular}

\section{Application of the font interface of the "Xue Xi Qiang Guo" APP}

Following the above-summarized design strategy of Song typeface, it designed some Song typeface effects that are more suitable for electronic screens ("Fig. 8"). It also uses the font interface of the "Xue Xi Qiang Guo" APP as the application object to realize the adaptive application of the electronic screen Song typeface. The "Xue Xi Qiang Guo" APP is supervised by the Publicity Department of the Communist Party of China. It is a high-quality platform based on all party members and facing the whole society based on $\mathrm{Xi}$ Jinping's new era of socialism with Chinese characteristics and the spirit of the 19th National Congress of the Communist Party of China. [4] The "Xue Xi Qiang Guo" APP uses Song typeface fonts for large fonts and boldface for small fonts. Visually, it is composed of the font size and thickness of Song typeface and boldface, respectively, combined with pictures to form the entire electronic reading interface. When reading, the reader jumps from the rhythmic Song typeface font on the previous line to the stylized boldface, from black font to gray font, which has a large visual jump and is prone to produce fatigue. It replaces the font on the interface with the optimized screen display Song typeface ("Fig. 9"). The experiment found that the small font size screen display Song typeface can be well displayed on the mobile phone display. The strokes of Song typeface are simplified in visual details due to the small font size, which happens to show the stylized effect of Song typeface, while the reconstruction of Song typeface with large font can tell the details, which has the distinctive characteristics of Song typeface. 
排版字号: $10.5 \mathrm{pt}$ 行距: $21 \mathrm{pt}$

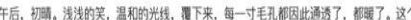

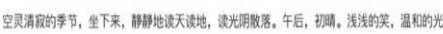

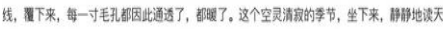

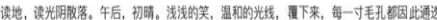

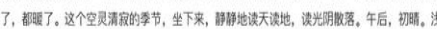

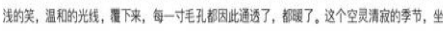

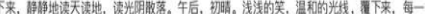

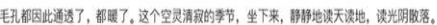

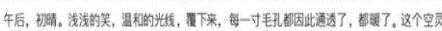

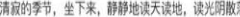

排牌量: $18 \mathrm{pt}$ 行距: $28 \mathrm{pt}$

午后, 初晴。浅浅的笑, 温和的光线, 覆下来, 每一寸毛 孔都因此通透了, 都暖了。这个空灵清寂的季节, 坐下来, 静 静地读天读地, 读光阴散落。午后, 初晴。浅浅的笑, 温和的 光线, 覆下来, 每一寸毛孔都因此通透了, 都暖了。这个空灵 清寂的季节, 坐下来, 静静地读天读地, 读光阴散落。午后, 初晴。浅浅的笑, 温和的光线, 覆下来, 每一寸毛孔都因此通 透了, 都暖了。这个空灵清寂的季节, 坐下来, 静静地读天读 地, 读光阴散落。

期肘字号: 27 pt 行距: 42 p

午后, 初晴。浅浅的笑, 温和的光线, 覆下来, 每一寸毛孔都因此通透了, 都暖 了。这个空灵清寂的季节, 坐下来, 静静地读天读地, 读光阴散落。午后, 初晴。浅浅 的笑, 温和的光线, 覆下来, 每一寸毛孔都因此通透了, 都暖了。这个空灵清寂的季

节, 坐下来, 静静地读天读地, 读光阴散落。

Fig. 8. Partial screen display optimization effect of Song typeface.

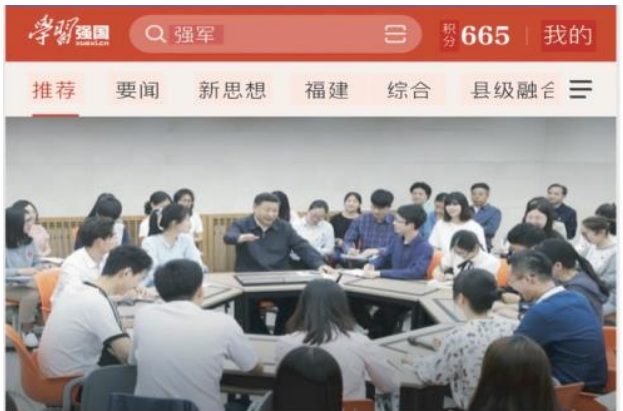

习近平与大学生

习近平宣布：“北斗三号卫星正式开通！”

新华视点 2020-07-3

习近平主持中共中央政治局会议 决定10月召开 党的十九届五中全会

“学习强国”学习平台 2020-07-30

中共中央召开党外人会士议 习近平主持宣布

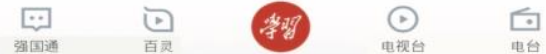

Fig. 9. Screen display application effect of Song typeface in "Xue Xi Qiang Guo" APP.

\section{CONCLUSION}

Characters were born from people's needs, and with the innovation of carriers, they continue to evolve to better meet people's needs. This paper introduces adaptive design theory, the purpose of which is to improve the state of Song typeface's inadaptation to screen display, improve the recognition and legibility of Song typeface in the screen display, and improve readers' reading experience. Science and technology are constantly advancing, the media is constantly being updated, the virtual display technology has begun to appear, and fonts will also face new challenges. This is a process that requires long-term practice and run-in period. Just as for thousands of years, printing and characters have continued to fit together.

\section{References}

[1] Ye Weidong. Research on urban grid design based on adaptability [D]. Southeast University, 2015. (in Chinese)

[2] Mai Minhua. Discover the beauty of digital reading [J]. Library Work and Study, 2011(8): 98-101. (in Chinese)

[3] Li Yujun. The Chinese cultural characteristics of Song typeface characters [J]. Decoration, 2005(10):116-117. (in Chinese)

[4] The Chongqing learning platform of "Xue Xi Qiang Guo" is online. Xinhuanet [reference date 2019-03-21] (in Chinese)

[5] Li Zhenyu, Wang Anxia. Comparative study on the legibility of the stroke design of Song typeface and Times New Roman [J]. Packaging Engineering, 2018,39(12):63-67.(in Chinese)

[6] Li Gang, Song Chenrui. Thoughts on the dimensions of the character design of the bamboo slips of Chu State in Baoshan during the Warring States Period - the correlation between the rule of ending a stroke, the outline and the centre of balance $[\mathrm{J}]$. Decoration, 2017(06):82-84.(in Chinese)

[7] Feng Weili, Jiang Yu. On the development of font design under new media [J]. Art Appreciations, 2020(02):40-41.(in Chinese) 